\title{
Three-dimensional periodontal investigations using a prototype handheld ultrasound scanner with spatial positioning reading sensor
}

\author{
Radu Chifor ${ }^{1,2}$, Mengxun $\mathrm{Li}^{3,4}$, Kim-Cuong T Nguyen ${ }^{3,5}$, Tudor Arsenescu' ${ }^{2}$, Ioana Chifor ${ }^{1,2}$, \\ Alexandru F Badea ${ }^{6}$, Mindra E Badea ${ }^{1}$, Mircea Hotoleanu ${ }^{7}$, Paul W Major ${ }^{8}$, Lawrence $\mathbf{H}$ \\ $\mathrm{Le}^{3,5,8}$
}

${ }^{1}$ Department of Preventive Dentistry, Faculty of Dental Medicine, "Iuliu Hatieganu” University of Medicine and Pharmacy, Cluj-Napoca, Romania, ${ }^{2}$ Chifor Research SRL, Cluj-Napoca, Romania, ${ }^{3}$ Department of Radiology and Diagnostic Imaging, University of Alberta, Edmonton, AB, Canada, ${ }^{4}$ Department of Implantology, School and Hospital of Stomatology, Wuhan University, Wuhan, China, ${ }^{5}$ Department of Biomedical Engineering, University of Alberta, Edmonton, AB, Canada, ${ }^{6}$ Anatomy and Embryology, Faculty of General Medicine, "Iuliu Hatieganu" University of Medicine and Pharmacy, Cluj-Napoca, Romania, ${ }^{7}$ Romanian Institute of Science and Technology, Cluj-Napoca, Romania, ${ }^{8}$ School of Dentistry, University of Alberta, Edmonton, AB, Canada

\begin{abstract}
Aim: To demonstrate the feasibility of the 3D ultrasound periodontal tissue reconstruction of the lateral area of a porcine mandible using standard 2D ultrasound equipment and spatial positioning reading sensors. Material and method: Periodontal $3 \mathrm{D}$ reconstructions were performed using a free-hand prototype based on a 2D US scanner and a spatial positioning reading sensor. For automated data processing, deep learning algorithms were implemented and trained using semi-automatically segmented images by highly specialized imaging professionals. Results: US probe movement analysis showed that non-parallel 2D frames were acquired during the scanning procedure. Comparing 3 different 3D periodontal reconstructions of the same porcine mandible, the accuracy ranged between $0.179 \mathrm{~mm}$ and $0.235 \mathrm{~mm}$. Conclusion: The present study demonstrated the diagnostic potential of 3D reconstruction using a free-hand 2D US scanner with spatial positioning readings. The use of automated data processing with deep learning algorithms makes the process practical in the clinical environment for assessment of periodontal soft tissues.
\end{abstract}

Keywords: periodontal ultrasonography; 3D imaging; artificial intelligence; ultrasound imaging; periodontal diagnosis

\section{Introduction}

Periodontal probing is the accepted gold-standard approach for the assessment of the periodontal soft-tissue [1]. Noninvasive techniques to image gingival soft tissues are not currently available in dentistry. Bi-dimensional ultrasound (2D US) imaging has already shown great potential for evaluating periodontal soft-tissue structures [2-5]. Lin et al demonstrated that periodontal probing depths could be measured with photoacoustic imaging

Received 23.09.2020 Accepted 18.02.2021

Med Ultrason

2021, Vol. 23, No 3, 297-304

Corresponding author: Ioana Chifor

31 Avram Iancu street,

400083, Cluj-Napoca, Romania

Phone: +40744117409

E-mail: ioana.chifor@umfcluj.ro
[6]. The imaging modality was more precise compared with periodontal probing, offered higher resolution images and covered all areas of the tooth. The gingival thickness could also be easily measured using ultrasound [7].

However, 2D periodontal US is not commonly used because clinicians have to imagine the three-dimensional (3D) view of the anatomic structures within the tissue volume from the planar US images [8]. Mechanical 3D probes are relatively compact and are convenient to operate, though they are larger than conventional linear probes. Their data acquisition and reconstruction times are short, which allows the viewing of high-quality 3D images in real-time. Volumetric US systems can fully capture a $3 \mathrm{D}$ region of interest, but current $3 \mathrm{D}$ systems require specialized transducers which are prohibitively expensive for many clinical departments and do not register image orientation with respect to the patient. Clini- 
cians are required to hold the mechanical 3D probes statically while acquiring images, which can lead to latent errors for data acquisition [9].

Developing smart computer algorithms to reconstruct 3D data volume using 2D frames could overcome problems related to 2D US imaging such as low reproducibility and operator dependency. Moore et al used a photoacoustic US transducer connected to a stepper motor for axial scanning and a sliding positioner for lateral control to obtain 3D images [10].

For 3D US imaging, besides the positioning and orientation errors that may corrupt the reconstruction process, another source of operator's dependency is image interpretation. Compared with other medical images (Xray, CT, MRI), the US images are characterized by higher noise, image contrast dependent on tissue density and low reproducibility. The interpretation is time consuming and is strongly dependent on clinician's experience. To overcome these challenges, various semi-automatic and automatic techniques to analyze the US images have been developed. The traditional approach was to use hand-crafted image processing techniques, such as edge detection, contrast enhancement, or line fitting $[11,12]$.

The development of deep learning models for computer vision has changed the paradigm for automatic US image processing and analysis. Deep learning is a powerful tool for extracting nonlinear features from data. This is particularly promising in US imaging, where predictable acoustic patterns are typically neither obvious nor easily hand-engineered [13].

To obtain the spatial orientation of the acquired 2D US periodontal images with respect to the patient and to generate 3D US model reconstruction of a larger area such as a maxillary hemiarch, our team developed a handheld ultrasound scanner prototype based on a standard 2D US machine and a spatial positioning reading sensor. In this paper, we report the preliminary results of periodontal 3D reconstruction using the prototype. For automated data processing, deep learning algorithms were implemented and trained using semi-automatically segmented images by highly specialized imaging professionals. The main objective of this study was to demonstrate the feasibility of the 3D US periodontal tissue reconstruction of the lateral area of a porcine mandible. The secondary objective was the evaluation of the accuracy and reproducibility of the method.

\section{Material and method}

A 3D high frequency US imaging prototype based on a standard medical US scanner (Vinno 6, Suzhou, China) with a high frequency $(10-23 \mathrm{MHz})$ and small aperture

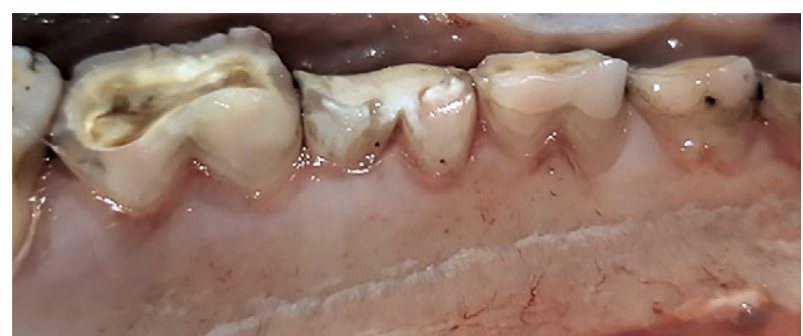

Fig 1. The porcine mandible showing the scanned area.

$(12.8 \mathrm{~mm})$ linear transducer (X10-23L, Vinno, Suzhou, China) and a positioning reading sensor embedded in an articulated measurement arm (Evo 7, RPS Metrology, Sona, Italy) was developed by Chifor Research SRL team and used for the study. The probe was attached to the sensor and the scanning was performed at $23 \mathrm{MHz}$. The positioning reading sensor tracked the trajectory of the transducer, providing coordinates and orientation during scanning as the output. The scanner had a free-hand architecture with 6 degrees of freedom. The transducer and the sensor were position calibrated and time synchronized at the beginning of the first scanning procedure. To test the accuracy and reproducibility of the prototype, three consecutive US scans (Scan 1, Scan 2 and Scan 3) were performed on the lingual side of 4 lateral teeth (two molars and two premolars) of a porcine mandible immersed in water (fig 1). The US examinations of the pig mandible were performed in January 2020 by the same operator. The mandible was harvested from a pig raised and slaughtered for alimentary purposes in farms organized according to European Union legislation and was not from experimental animals. The scans started between the third and second molars, moved in mesial direction to the second premolar and then moved backwards, in distal direction to the starting point. The US scanner had a frame rate of 30 frames or images per second; 500 consecutive frames from each scan were used for 3D reconstruction corresponding to the lateral area of the mandible. A total number of 1500 2D periodontal US images were taken into consideration for the prototype accuracy evaluation. For each 2D frame, the spatial and orientation information was utilized to organize the frame in the 3D space.

\section{Semi-automatic segmentation}

Prior to the $3 \mathrm{D}$ reconstruction, the $2 \mathrm{D}$ images were segmented for three types of tissues, i.e., tooth, bone and gingiva. The 2D images of Scan 1 were annotated and segmented by the research team at the University of Alberta using the in-house developed labeling software. Figure 2 shows the workflow of the segmentation process to segment the alveolar bones automatically. An oral US imaging specialist or reader initiated a graph-cut based segmentation process by roughly defining a region inside 


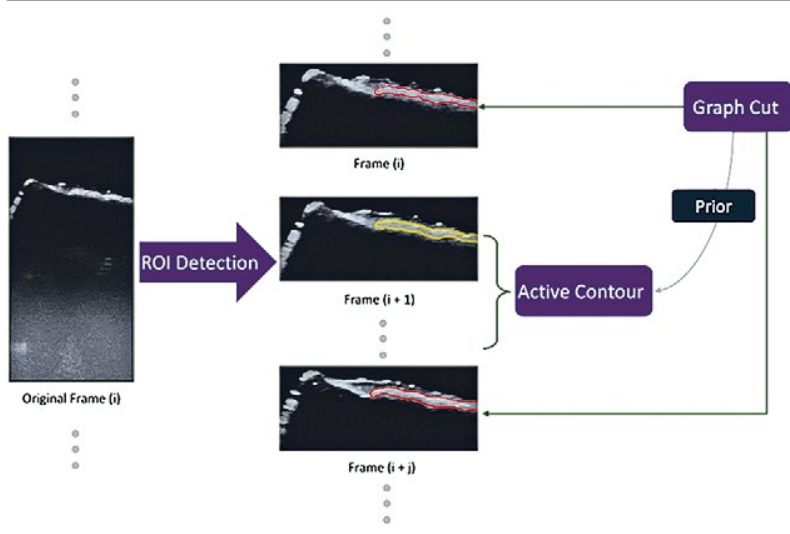

Fig 2. The segmentation workflow. The red and yellow marked contours, representing the cortical bones, were labeled by the semi-automatic and fully automatic methods, respectively.

the desired structures in selected frames to serve as seed pixels. This process started off in a frame and was skipped for the next few frames if the consecutive frames had similar structures. It was then followed by an automatic contour interpolation and refinement process, which used an active contour model to incorporate the intensity and shape prior to information from the previous step. The reader checked the interpolation result and revised the labeling manually with the built-in "brush" and "eraser" tools to ensure accurate segmentation for every frame.

To extract the Region of Interest (ROI), representing the marginal periodontal tissue area (cortical bone, tooth crown and gingival tissue) and to exclude the other part of the image, the integral projection along the horizontal axis of the images upon the depth axis was performed and modified by multiplying the integral curve with a one-side Tukey taper, which did not alter the early portion of the curve but suppressed the tail of the integration. The resultant was a bimodal signal which represented the ROI and the structure-free region, respectively. The Otsu's thresholding method [14] was then used to find the optimum position separating these two peaks to extract the ROI.

The simple linear iterative clustering (SLIC) technique [15] was applied to the ROI image to group pixels with the same characteristics into superpixels for segmentation. Following Boykov et al [16], a graph-cut method was implemented to segment tissues using the seed pixels selected by the reader. The distance penalties were added to the regional cost comparing to the original graph-cut method proposed by Boykov et al [16], which was based on the observation that intensity distribution and gradient alone could not sufficiently generate accurate segmentation in our case.

The graph-cut segmentation was performed for a few frames and then the intermediate similar frames were automatically interpolated. An active contour model [17] was used to incorporate the shape and intensity prior information from the previous semi-automatic segmentation step to perform the interpolation. The in-house developed GUI software for semi-automatic segmentation was implemented in the Python programming language, based mainly on libraries such as OpenCV, Numpy, and Tkinter. The pyMaxflow library [18] was also employed to optimize the Max-flow/Min-cut algorithm for the graph-cut [17].

\section{Automatic segmentation}

Images from Scan 2 and Scan 3 were segmented by the research team at the Romanian Institute of Science and Technology using Neural Networks Algorithms and a deep learning model for object detection and image segmentation developed by extending the Mask R-CNN model $[19,20]$. The model was trained on an Intel® ${ }^{\circledR}$ Core $^{\mathrm{TM}}$ i7-9700K CPU@ $3.60 \mathrm{GHz} \times 8$ cores, 32GB RAM computer equipped with GeForce RTX NVidia GPU with 8GB RAM. The 500 annotated US images from Scan 1 were used for training. The annotation consists of separate images that contain the masks for 3 types of tissue: tooth, bone, and gingiva. This model was preloaded with the COCO weights [21] and was trained using the images from Scan 1. The training dataset was created by randomly selecting 420 out of 500 images from Scan 1. The other 80 images were used for model validation. The training was done on one GPU (CUDA) processing simultaneously 4 images. All Mask R-CNN weights were retrained to detect 3 new classes by running 5 epochs and 150 steps per epoch. As a result, the trained model was able to automatically detect and segment the three types of tissues.

\section{$3 D$ reconstruction and statistical analysis}

Data acquisition and 3D reconstruction were performed by the team at Chifor Research SRL using the 3D US scanner prototype and the developed software. After the US data was acquired, each frame was paired or matched with the sensor's readings. The spatial coordinates and orientation of each frame were determined. All the frames were then re-organized in the $3 \mathrm{D}$ space, thus reconstructing the $3 \mathrm{D}$ data volume. The Cloud Compare Open-Source Software (CCOSS) Version 2.6.3 was used to compare the $3 \mathrm{D}$ reconstructed frames after each scan. Standard deviation (std) and mean distances were calculated between the point clouds.

\section{Results}

Table I summarizes the pertinent information about the three scans and the reconstructed data volume.

Using Scan 1 readings as example, the overall translation movement (fig $3 \mathrm{a}$ ) shows that the US probe moved 
$3 D$ periodontal investigations using a prototype handheld US scanner with spatial positioning reading sensor

Table I. Scanning time and spatial positioning readings for the three ultrasound scans

\begin{tabular}{|c|c|c|c|}
\hline & Scan 1 & Scan 2 & Scan 3 \\
\hline Scanning time (sec) & 16.597 & 16.584 & 16.585 \\
\hline Changing main direction after $(\mathrm{sec})$ & 9.758 & 10.345 & 11.206 \\
\hline \multicolumn{4}{|l|}{ Dimension of the examined volume (mm) } \\
\hline $\mathrm{X}$ & 49.114 & 51.551 & 63.316 \\
\hline $\mathrm{Y}$ & 25.446 & 24.434 & 24.180 \\
\hline $\mathrm{Z}$ & 8.476 & 11.131 & 5.673 \\
\hline \multicolumn{4}{|c|}{ Number of transducer direction changes during scanning } \\
\hline X $\quad$ Mesio - Distal (toward mesial) & 22 & 12 & 15 \\
\hline (toward distal) & 23 & 11 & 15 \\
\hline Corono - Apical (toward mesial) & 41 & 47 & 33 \\
\hline (toward distal) & 42 & 48 & 33 \\
\hline \multirow{2}{*}{$\begin{array}{l}\text { Z Buccal - Lingual (toward mesial) } \\
\text { (toward distal) }\end{array}$} & 43 & 47 & 40 \\
\hline & 44 & 47 & 41 \\
\hline
\end{tabular}

in a well-defined plane in one direction from distal to mesial from the $1^{\text {st }}$ frame to the $295^{\text {th }}$ frame and then in the opposite direction from mesial to distal from $296^{\text {th }}$ to $500^{\text {th }}$ frame. The relative movement of the probe between frames (fig 3b) shows the short distance pattern where the spikes represent changes in the probe movement direction between two adjacent frames. The figure also shows the long-distance movement pattern from molar 3 towards premolar 2 with two major changes in direction along the overall trajectory of the probe. While moving along the posterior-anterior direction away from the starting point, the majority of the motion are in the positive range from $1^{\text {st }}$ to $295^{\text {th }}$ frame. During the anterior-posterior motion, the probe moved in the opposite direction and therefore, from $296^{\text {th }}$ until $500^{\text {th }}$ most values are negative. Figure $3 \mathrm{c}$ shows the angular variations of the probe between neighboring frames, where the spikes represent changes in plane angulation movement, showing that non-parallel 2D frames were acquired during the scanning procedure.

Five hundred US images from Scan 1, which included the tooth, alveolar bone and gingiva, were segmented semi-automatically. The average speed for the semi-automatic segmentation algorithm was around 45 seconds per image. These images were scaled and converted to a specific binary format compatible with a Mask R-CNN deep learning model. Four hundred and twenty images were used as a training set and the rest of the images as a validation set. The accuracy of the training and validation sets was evaluated by the mean accuracy precision mAP [20]. For 5 epochs with 150 steps and batch size of 4 , the training time was 2 hours and 40 minutes, and mAP was over $90 \%$. At the pixel level, the precision between the annotated and detected masks was calculated as IoU (Intersection over Union). The average IoU was $85 \%$. Scan 2 and Scan 3 were segmented using the trained model from Scan 1. All three types of tissues were identified in all images from Scan 2 and Scan 3. The precision of the automated segmentation was evaluated globally by comparing the results with those obtained in Scan 1. An example of the segmentation results is shown in Figure 4. The automatic segmentation took 0.45 second per image.

The 3D reconstruction of the teeth for the three scans is shown in Figure 5. Grey scale intensity values of the voxels from the point clouds were converted to RGB

a)
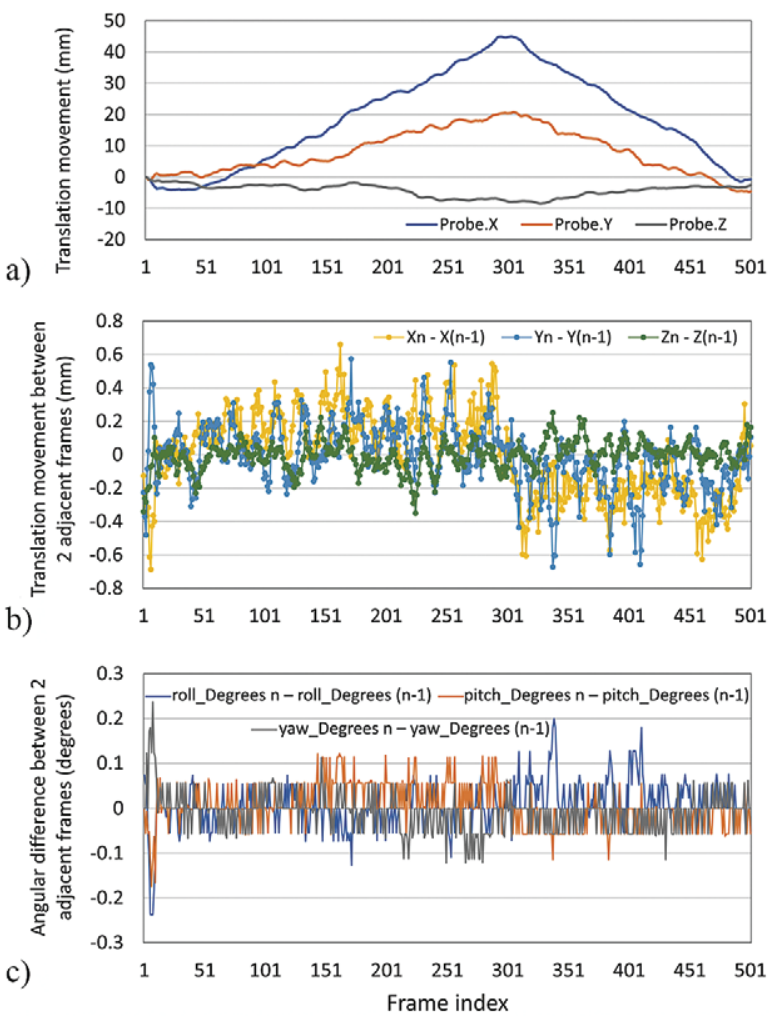

Fig 3. The spatial position and orientation readings of the US probe for Scan 1. (a) The translational motion; (b) The relative translational motion between frames; (c) The relative angular variations of the probe between frames. 

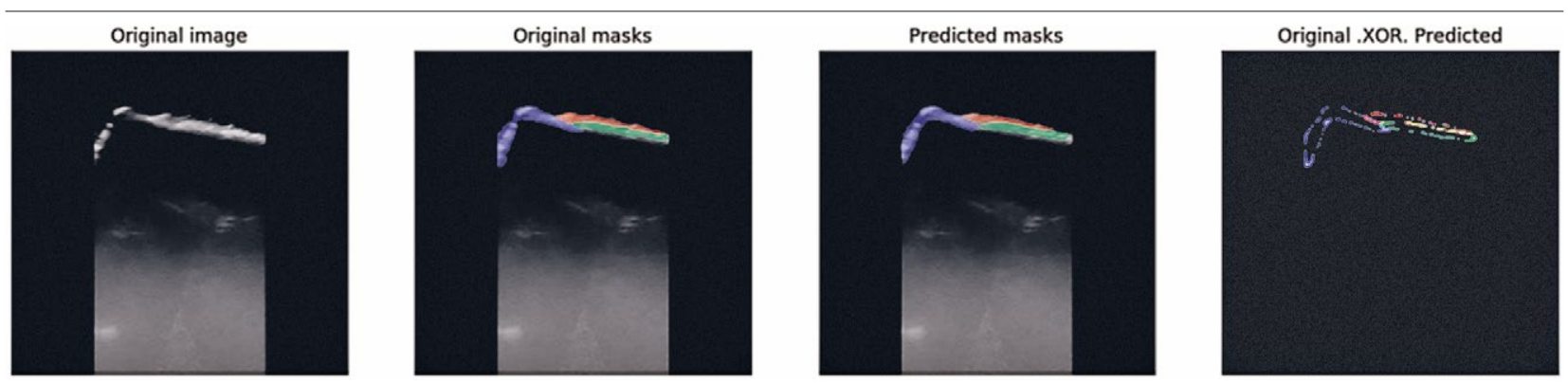

Fig 4. Image segmentation: (a) the original US image, (b) the semi-automatic segmentation of the image, (c) the automatic segmentation of the image, and (d) the difference between semi-automatic and automatic segmentations.

(red, green, blue) values for better visualization. The color conversion and visualization of the point clouds were performed using CCOSS. Six arbitrary points (R0, $\mathrm{R} 1, \ldots, \mathrm{R} 5)$ and six corresponding points $(\mathrm{A} 0, \mathrm{~A} 1, \ldots$, A5) were selected by the operator in the reconstructed Scan 1 and Scan 2 areas, respectively. Using these points, CCOSS aligned the point clouds for statistical analysis to determine the mean distance and the standard deviation between them (fig 6).

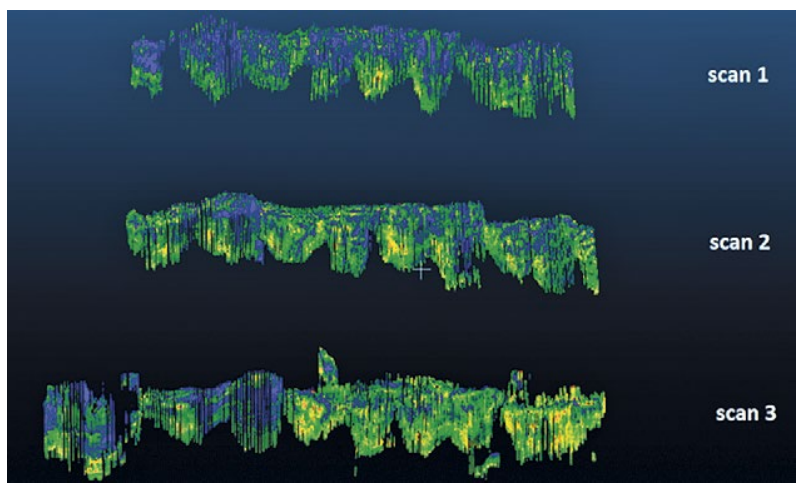

Fig 5. 3D reconstruction of the teeth for the three scans.

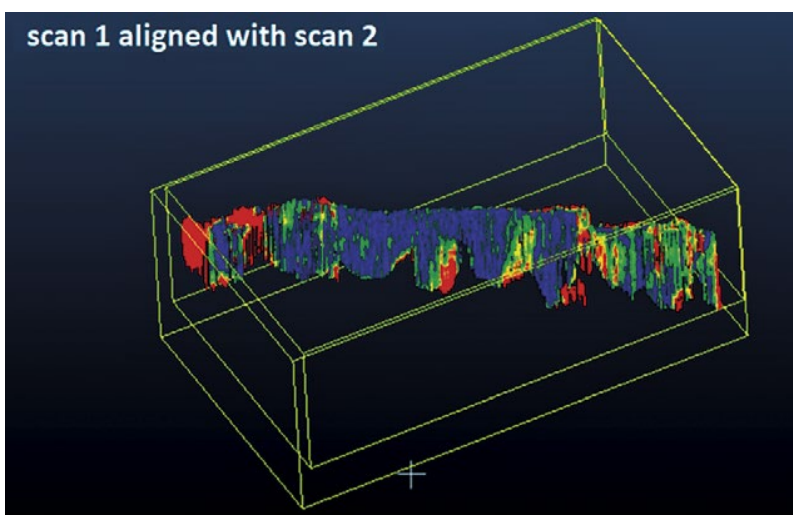

Fig 7. The alignment of the two reconstructions (Scan 2 and Scan 1) where Scan 1 is taken as reference point cloud. The colors correspond to the distances between the two point clouds. Red is for larger distances, blue is for smaller distances, and green is for medium distances.
Figure 7 shows the aligned 3D reconstruction of the teeth from Scan 2 and Scan 1. The 3D reconstruction was colored according to the distances between the 2 point clouds in figure 6 . The mean distance and standard deviation between the points from the two scans (at tooth level) are 0.179 and $0.187 \mathrm{~mm}$, respectively. Similarly, figure 8 shows the aligned reconstruction of the teeth from Scan 3 and Scan 1. Scan 3 included an extra tooth distally, which was not covered in Scan 1. The color an-

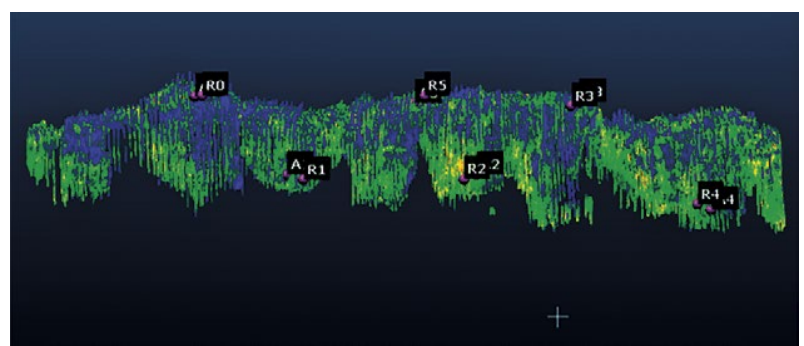

Fig 6. The alignment of the two scans (Scan 1 and Scan 2) where Scan 1 was used as reference. The grey scale pixel intensity values between 0 and 255 correspond to red, green, and blue values, respectively. Blue is for values closer to 0 , green is for intermediate values, and red is for values closer to 255 .

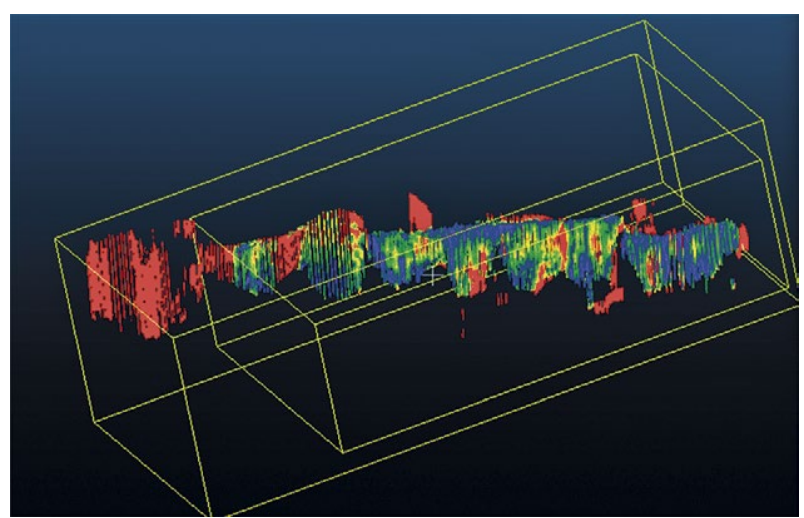

Fig 8. The alignment of the two reconstructions (Scan 3 and Scan 1) where Scan 1 is taken as reference point cloud. The colors correspond to the distances between the two point clouds. Red is for larger distances, blue is for smaller distances, and green is for medium distances. 


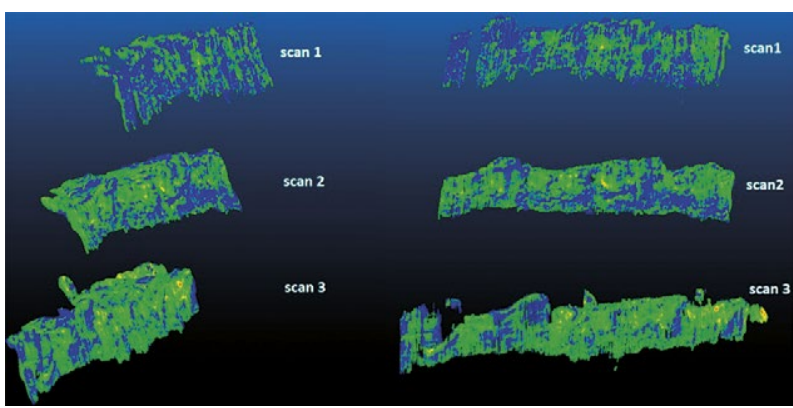

Fig 9. 3D reconstruction of the teeth at the tooth and gingiva levels.

notation is similar to figure 7. To eliminate the impact of the outlier points caused by the extra tooth, we excluded the points with distance larger than 500 microns during the statistical analysis. The mean distance and standard deviation between the points from the two scans (at tooth level) are 0.235 and $0.206 \mathrm{~mm}$, respectively. Figure 9 shows the $3 \mathrm{D}$ reconstruction at the teeth and gingival levels. Figure 10 shows the aligned reconstructions of the teeth and gingiva from Scan 2 and Scan 1. The mean distance and standard deviation between the points from Scan 2 and Scan 1 are 0.191 and $0.186 \mathrm{~mm}$, respectively.

\section{Discussion}

The challenge of segmenting the periodontal structures has three main aspects. First, low signal-to-noise ratio, low signal-to-contrast ratio and motion artifact blur the tissue boundaries. Second, different tissues share the same intensity distribution, i.e., the tooth and alveolar bone typically have similar intensity ranges. Also, the echoes from the surface of the gingiva could be strong, which leads to intensity values close to those of bone and tooth. Last, the shape of the structures varies in different sections of the dentition, especially in the transitional area of adjacent teeth. In this area, periodontal structures show significantly different patterns.

The semi-automatic or automatic segmentation of medical US images has been extensively reported in literature, and a review of those applications and techniques was performed by Meiburger et al [22]. However, there are limited reports on US image segmentation in dentistry. Pekam et al [23] used the model-based surface point matching segmentation method to acquire the surface of the prepared artificial teeth model to facilitate prosthetic manufacturing. Xu et al [24] traced the contour in the US tongue image sequence using the active contour method with an automatic re-initialization technique. Chifor et al [25] explored the usage of US in the detection of gingival sulcus with the assistance of active contour based semiautomatic sulcus segmentation in the swine specimen;

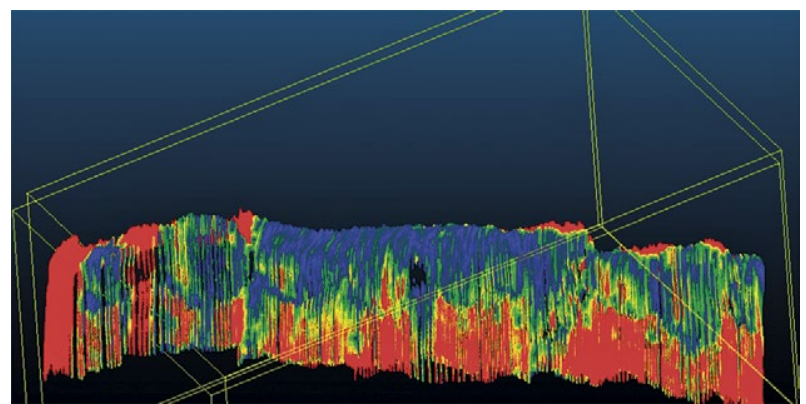

Fig 10. The alignment of the two reconstructions (Scan 2 and Scan 1) at the tooth and gingiva level, where Scan 1 is taken as a reference point cloud. The colors correspond to the distances between the two point clouds. Red is for larger distances, blue is for smaller distances, and green is for medium distances.

Nguyen et al [26] segmented alveolar bone in US images using the graph-cut based method with mandibular incisors from piglets, and subsequently adopted a deep learning approach to detect alveolar bone in humans [4].

The major limitation of our semi-automatic segmentation method was the long segmentation time (45 seconds per frame). To overcome this drawback, a deep learning model for object detection and image segmentation was developed. After training, the algorithm was able to automatically detect and segment the three types of tissues. The developed machine learning based segmentation algorithm was a two-order of magnitude faster. It can segment one image in 0.45 second. At the same time, the algorithm demonstrated high accuracy with all tissues identified, and $85 \%$ of the segmented area corresponds to that identified by the semi-automated algorithm.

The accuracy of our model is at the same level or above the average of results presented in literature [2729]. The average accuracy from 25 independent studies published in a recent systematic review, is $88.6 \%$ for sensitivity and $93.9 \%$ for specificity [30]. However, the reported study included all types of medical imaging modalities (radiography, CT, US) applied to various body regions, while our algorithm used only US, which is known to be more difficult to interpret. Another publication on algorithms for US images of muscle only reported an accuracy of $76 \%$ [31]. When the discussion is restricted to the maxillofacial region, the reported deep learning algorithms were applied mostly to radiological and CT imaging. The accuracy of these techniques ranges from $80 \%$ [32] to $87 \%$ [33]. Rana et al [34] reported an accuracy of about $75 \%$ when processing photographic dental images.

The 3D reconstruction discrepancy in the present study compared with other studies is lower. Marotti et al also used CCOSS to calculate the mean distance and reported a reconstruction precision between 12.34 to $46.38 \mu \mathrm{m}$ [35]. In the Marotti et al [35] study, only one 
tooth was examined compared with 4 teeth examined in our study. In regard to the accuracy of CBCT, Tomasi et al reported $0.36 \mathrm{~mm}$ for the horizontally positioned mandible, $0.48 \mathrm{~mm}$ for the inclined mandible and an overall absolute mean measurement error of $0.40 \mathrm{~mm}$ [36], while our study rendered a better accuracy between $0.179 \mathrm{~mm}$ and $0.235 \mathrm{~mm}$.

Our paper has certain limitations: the examinations were performed on the same porcine mandible generating probably an overestimated accuracy for the predictions made using artificial intelligence algorithms. Also, it was observed that the prediction underestimated the area of the tissue (refer to Figure 4 for example), most probably due to the low number of training images. Further effort is needed in the future to improve the results using a higher number of highly accurate segmented US periodontal images for training and validation. Despite these limitations the results regarding the reproducibility of the 3D US reconstructions from consecutive scans, using our handheld prototype are promising, qualitatively and quantitatively. The main advantage of the proposed 3D ultrasound reconstruction method is the free-hand architecture of the 3D US scanner prototype, with which one can examine a full maxillary arch.

\section{Conclusions}

The present feasibility study demonstrated the diagnostic potential of 3D reconstruction using a free-hand 2D US scanner with spatial positioning readings. The use of automated data processing with deep learning algorithms makes the process practical in the clinical environment for the assessment of periodontal soft tissues. Further research should explore reconstruction algorithms with/without interpolation between frames to improve the accuracy and resolution of the reconstruction results. Proper display of the 3D images corresponding to tissue types is also important for the clinical application.

\section{Acknowledgements}

This study was partially realized with the material, equipment, technology, and logistic support of Chifor Research SRL through the project Periodontal ultrasonography in diagnosing and monitoring the periodontal disease - Chifor Research SRL, Operational Program Competitivity, Ministry of European Funds from Romania, P_38 930 12.10 .2017 . The collaborative work was partly supported by the Natural Sciences and Engineering Research Council of Canada (LHL), Alberta Innovates Technology Futures Doctoral Fellowship (KCTN), and Wuhan University Scholarship (MXL).
This paper was published under the frame of the European Social Fund, Human Capital Operational Program 2014-2020, project no. POCU/380/6/13/125171 and EIT Health - RIS Innovation Program 2020, project ID 2020 RIS-1001-8253.

\section{Conflict of interest: none}

\section{References}

1. Andrade R, Espinoza M, Gómez EM, Espinoza JR, Cruz E. Intra- and inter-examiner reproducibility of manual probing depth. Braz Oral Res 2012;26:57-63.

2. Chifor R, Badea AF, Chifor I, Mitrea DA, Crisan M, Badea ME. Periodontal evaluation using a non-invasive imaging method (ultrasonography). Med Pharm Rep 2019;92(Suppl No 3):S20-S32.

3. Nguyen KC, Le LH, Kaipatur NR, Zheng R, Lou EH, Major PW. High resolution ultrasonic imaging of dento-periodontal tissues using a multielement phased array system. Ann Biomed Eng 2016;44:2874-2886.

4. Nguyen KCT, Duong DQ, Almeida FT, et al. Alveolar Bone Segmentation in Intraoral Ultrasonographs with Machine Learning. J Dent Res 2020;99:1054-1061.

5. Nguyen KC, Pacheco-Pereira C, Kaipatur NR, Cheung J, Major PW, Le LH. Comparison of ultrasound imaging and cone-beam computed tomography for examination of the alveolar bone level: a systematic review. PLoS One 2018;13:e0200596.

6. Lin CY, Chen F, Hariri A, et al. Photoacoustic Imaging for Noninvasive Periodontal Probing Depth Measurements. J Dent Res 2018; 97:23-30.

7. Kloukos D, Koukos G, Doulis I, Sculean A, Stavropoulos A, Katsaros C. Gingival thickness assessment at the mandibular incisors with four methods: A cross-sectional study. J Periodontol 2018;89:1300-1309.

8. Huang Q, Zeng Z. A Review on Real-Time 3D Ultrasound Imaging Technology. BioMed Research International. Biomed Res Int 2017;2017:6027029.

9. Herickhoff CD, Morgan MR, Broder JS, Dahl JJ. Low-cost Volumetric Ultrasound by Augmentation of 2D Systems: Design and Prototype. Ultrason Imaging 2018;40:35-48.

10. Moore C, Bai Y, Hariri A, et al. Photoacoustic imaging for monitoring periodontal health: A first human study. Photoacoustics 2018;12:67-74.

11. Li Z, Zhu P, Toyomura T, Noguchi Y. Automatic Image Analysis and Recognition for Ultrasound Diagnosis and Treatment in Cardiac, Obstetrics and Radiology. Intechopen, 20184 July, doi:10.5772/intechopen.76284.

12. Lu X, Georgescu B, Zheng Y, Otsuki J, Comaniciu D. Automated detection of planes from three-dimiensional echocardographc data. 2011 Dec; US Patent 8073215B2.

13. Brattain LJ, Telfer BA, Dhyani M, Grajo JR, Samir AE. Machine learning for medical ultrasound: status, methods, and future opportunities. Abdom Radiol (NY) 2018;43:786-799.

14. Otsu N. A threshold selection method from gray-level histograms. IEEE Trans Syst Man Cybern 1979;9:62-66. 
304 Radu Chifor et al 3D periodontal investigations using a prototype handheld US scanner with spatial positioning reading sensor

15. Achanta R, Shaji A, Smith K, Lucchi A, Fua P, Susstrunk S. SLIC Superpixels. June 2010; EPFL Technical Report 149300.

16. Boykov YY, Jolly MP. Interactive graph cuts for optimal boundary \& region segmentation of objects in N-D images. Proceedings Eighth IEEE International Conference on Computer Vision 2001;1:105-112.

17. Gao H, Chae O. Individual tooth segmentation from CT images using level set method with shape and intensity prior. Pattern Recognit 2010;43:2406-2417.

18. Boykov Y, Kolmogorov V. An experimental comparison of min-cut/max-flow algorithms for energy minimization in vision. IEEE Trans Pattern Anal Mach Intell 2004;26:11241137.

19. He K, Gkioxari G, Dollár P, Girshick R. Mask R-CNN. IEEE International Conference on Computer Vision (ICCV) Oct. 22-29, 2017:. Venice, Italy.

20. Abdulla W. Mask R-CNN for object detection and instance segmentation on Kerasand TensorFlow. 2017. Available at: https://github.com/matterport/MaskRCNN.

21. Lin TY, Maire M, Belongie SJ, et al. Microsoft COCO: Common Objects in Context. In: Fleet D, Pajdla T, Schiele B, Tuytelaars T. (Eds.). Computer Vision ECCV 2014:740-755. doi:10.1007/978-3-319-10602-1_ 48

22. Meiburger KM, Acharya UR, Molinari F. Automated localization and segmentation techniques for b-mode ultrasound images: A review. Comput Biol Med 2018;92:210 235.

23. Chuembou Pekam F, Marotti J, Wolfart S, Tinschert J, Radermacher K, Heger S. High-frequency ultrasound as an option for scanning of prepared teeth: an in vitro study. U1trasound Med Biol 2015;41:309-316.

24. Xu K, Yang Y, Stone M, et el. Robust contour tracking in ultrasound tongue image sequences. Clin Linguist Phon 2016;30:313-327.

25. Chifor R, Badea ME, Mitrea DA, et al. Computer-assisted identification of the gingival sulcus and periodontal epithelial junction on high-frequency ultrasound images. Med Ultrason 2015;17:273-279.

26. Nguyen KCT, Shi D, Kaipatur NR, et al. Graph cuts-based segmentation of alveolar bone in ultrasound imaging. 2018. IEEE International Conference on Bioinformatics and
Biomedicine (BIBM). Madrid, Spain, 2018:2049-2055. doi:10.1109/BIBM.2018.8621309.

27. Karatas $\mathrm{OH}$, Toy E. Three-dimensional imaging techniques: A literature review. Eur J Dent 2014;8:132-140.

28. Mohan R, Singh A, Gundappa M. Three-dimensional imaging in periodontal diagnosis - Utilization of cone beam computed tomography. J Indian Soc Periodontol 2011;15:11-17.

29. Kulczyk T, Rychlik M, Lorkiewicz-Muszyńska D, AbreuGłowacka M, Czajka-Jakubowska A, Przystańska A. Computed Tomography versus Optical Scanning: A Comparison of Different Methods of 3D Data Acquisition for Tooth Replication. Biomed Res Int 2019;2019:4985121.

30. Liu X, Faes L, Kale AU, et al. A comparison of deep learning performance against health-care professionals in detecting diseases from medical imaging: a systematic review and meta-analysis. Lancet Digit Health 2019;1:e271-e297.

31. Burlina P, Billings S, Joshi N, Albayda J. Automated diagnosis of myositis from muscle ultrasound: Exploring the use of machine learning and deep learning methods. PLoS One 2017;12:e0184059.

32. Lee JH, Kim DH, Jeong SN, Choi SH. Diagnosis and prediction of periodontally compromised teeth using a deep learning-based convolutional neural network algorithm. J Periodontal Implant Sci 2018;48:114-123.

33. Hiraiwa T, Ariji Y, Fukuda M, et al. A Deep-Learning Artificial Intelligence System for Assessment of Root Morphology of the Mandibular First Molar on Panoramic Radiography. Dentomaxillofac Radiol 2019;48:20180218.

34. Rana A, Yauney G, Wong LC, Gupta O, Muftu O, Shah P. Automated Segmentation of Gingival Diseases from Oral Images. 2017. IEEE Healthcare Innovations and Point of Care Technologies (HI-POCT), Bethesda, MD, 2017:144147. doi:10.1109/HIC.2017.8227605.

35. Marotti J, Broeckmann J, Chuembou Pekam F, Praça L, Radermacher K, Wolfart S. Impression of subgingival dental preparation can be taken with ultrasound. Ultrasound Med Biol 2019;45:558-567.

36. Tomasi C, Bressan E, Corazza B, Mazzoleni S, Stellini E, Lith A. Reliability and reproducibility of linear mandible measurements with the use of a cone-beam computed tomography and two object inclinations. Dentomaxillofac Radiol 2011;40:244-250. 